\title{
Endovascular management of recurrent anterior communicating aneurysm previously embolized. Case presentation
}

\author{
A. Chiriac ${ }^{1,2}$, Georgiana Ion ${ }^{1,2}$, Z. Faiyad ${ }^{1,2}$, I. Poeata ${ }^{1,2}$ \\ 1 "Grigore T. Popa" University of Medicine and Pharmacy, lasi, \\ ROMANIA \\ 2 "Prof. Dr. N. Oblu" Clinic Emergency Hospital, Iasi, RomanIA
}

\begin{abstract}
Although endovascular coiling treatment has been widely accepted as the method of choice for intracranial aneurysms, concerns about its durability are still discussed. Attention was largely focused on aneurysm recurrence after coil occlusion with possible unfavourable evolution to a new bleeding episode. We present our experience of a patient with a ruptured anterior communicating artery aneurysm previously treated by endovascular coil embolization that presented over a 4-year period for aneurysm recurrence.
\end{abstract}

\section{INTRODUCTION}

Coil embolization for intracranial aneurysms is currently the most accepted modality of treatment for patient with this vascular pathology. This is due to numerous studies which demonstrated its effectiveness in preventing rebleeding after aneurysmal rupture and a better outcome in terms of disability-free survival compared with aneurysmal neck clipping. Today, the main concern in endovascular treatment is represented by its durability due to aneurysm recurrence with disastrous potentially for the patient.

The purpose of this study was to describe a clinical situation requiring repeat embolization in a patient with a ruptured anterior communicating artery aneurysm previously treated by endovascular coil embolization, and discuss different aspects of aneurysm recurrence.

\section{CASE PRESENTATION}

A 50-year-old woman was referred for evaluation of an embolized anterior communicating aneurysm that demonstrated small coil compaction by fundus migration and aneurysm regrowth on serial angiography control. Four years before this referral, she had undergone an endovascular coil occlusion for a ruptured anterior communicating aneurysm. The patient made a good recovery.

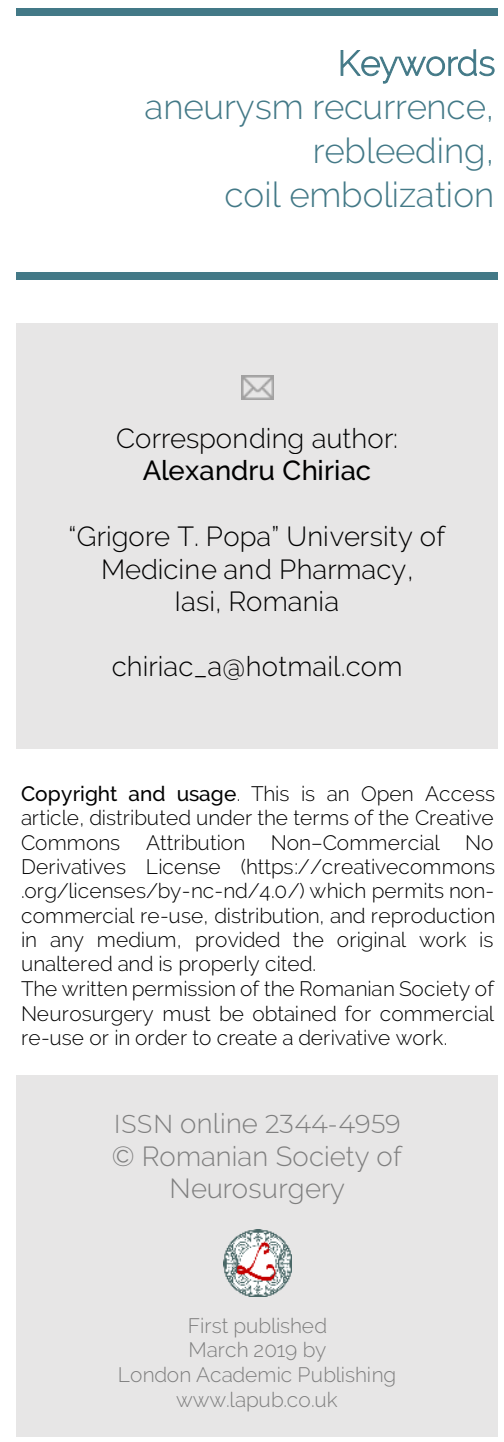


Postoperative angiography control at 12 months revealed a small aneurysm neck repermeabilization due to partial coil compaction. The angiography control at 2 and 3 years showed a slow development of the remnant neck portion by aneurysm regrowth. Initially, a conservative management by imaging monitoring was decided. The angiography control at 4-year interval revealed a clear aneurysm sac enlargement with a 1:1 ratio of occluded aneurysm versus the permeable portion of aneurysm sac. A new session of coil embolization was decided for complete occlusion of the residual aneurysm (Fig.1).
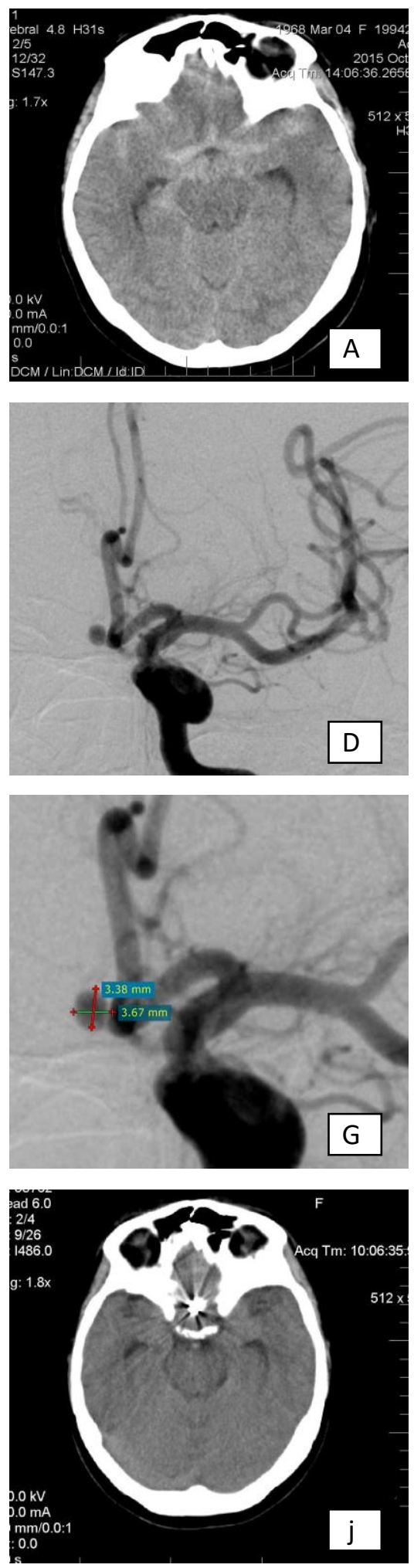
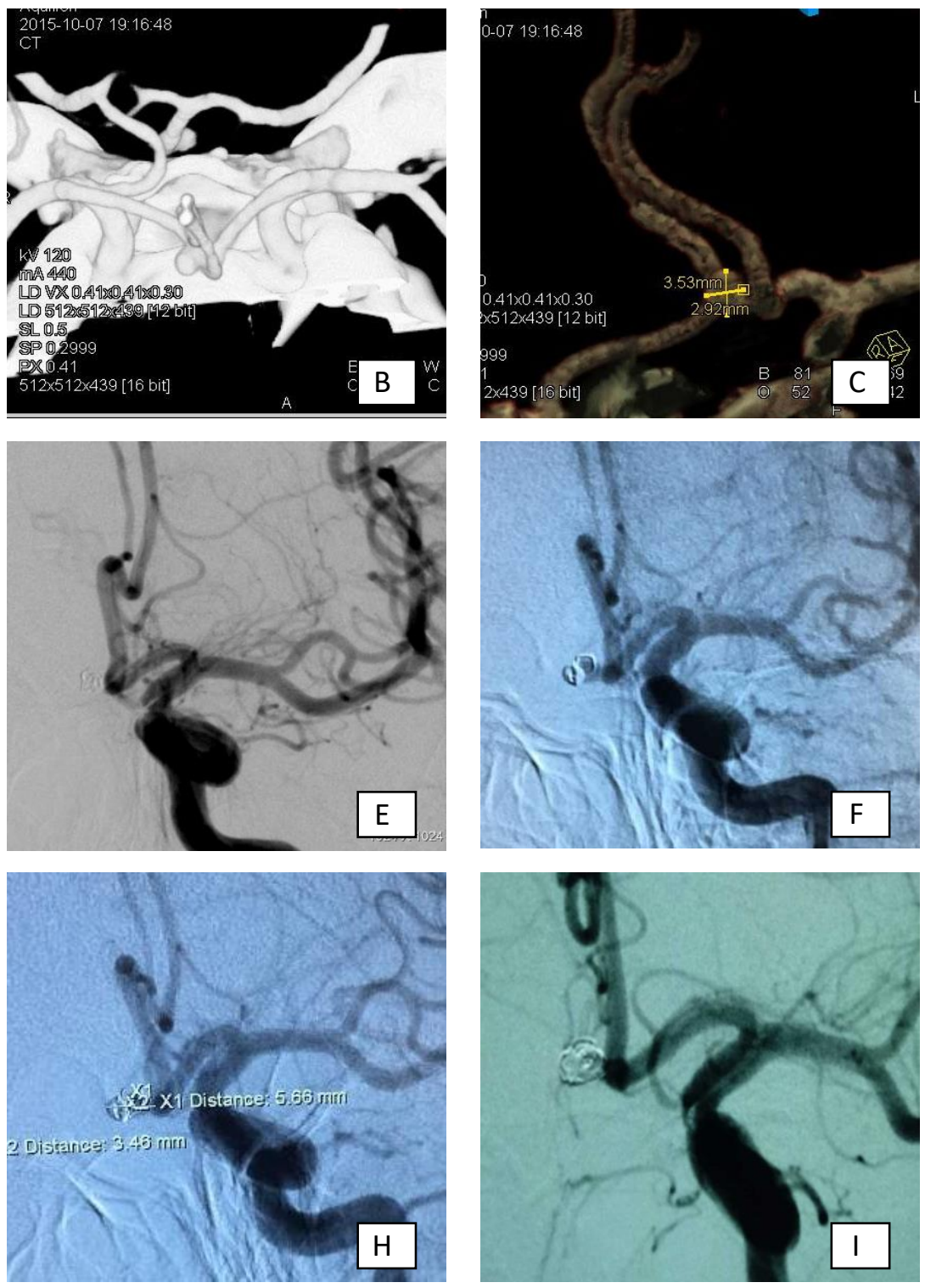

FIGURE 1: A-Brain CT scan showing the initial SAH; B, C-Angio-CT diagnosis of ACoA aneurysm and initial measurements; D, G-Initial DSA diagnostic and aneurysm measurements; E-DSA control postembolization with coils; F, H- DSA control 4 years later showing aneurysm regrowth; Ipostprocedural DSA control after additional coil embolization; J- Brain CT scan control after additional coil embolization. 


\section{Endovascular Procedure}

The interventional neurosurosurgeon team performed the endovascular treatment with the patient under general anaesthesia in a monoplane angiosuite. Treatment approach was performed through the right femoral access route with a $6 \mathrm{~F}$ introduction sheath. Anticoagulation with $5000 \mathrm{IU}$ heparin was used from the beginning of the procedures according to our centre protocol for unruptured aneurysm. The systemic administration of heparin is continued to entire period of the procedure (perfuzable solution of $1 \mathrm{ml}$ Heparin $/ 500 \mathrm{ml} \mathrm{NaCl}$ ). A $6 \mathrm{~F}$ guiding catheter (Ghider Softip 40XF Boston Scientific) was placed in the left internal carotid artery that provided arterial supply to the aneurysm remnant. Multiple DSA series were obtained in different degrees of obliquity. Also, a three-dimensional rotational angiography was finally performed to choose the working projection. Once an appropriate angle of obliquity was obtained on 3D PostProcessing unit that optimally highlight the relationship of neck of the aneurysm remnant and the parent vessel, the position was automatically send it to angiograph C-Arm. On this projection a road-mapping acquisition is performed and maintained throughout the duration of the procedure. A microcatheter (Excelsior SL-10, Stryker) was then advanced over a microguidewire (Transend 0.0014, Boston Scientific) under road-mapping guidance into the aneurysm remnant. Two GDCs-10 (Stryker) were then advanced and detached in the aneurysm remnant. The attempt to introduce the third GDC was not technically possible due to its distal end protruding into the parent vessel. Serial DSAs were performed to verify and to monitor the progress of aneurysm remnant occlusion. Once the aneurysm remnant was deemed to be satisfactorily occluded, the microcatheter was gently removed. This was followed by the guiding catheter removal through the femoral sheaths after obtaining a final DSA. The femoral sheaths were then removed from the femoral artery and hemostasis was obtained by prolonged compression. The patient was awakened from general anaesthesia and then admitted to the neuro-intensive care unit for 24-hour observation and monitoring. Patient was discharged home 48 hours later. Antiplatelet medication was maintained for the period of hospitalization.

FOLLOW-UP PROTOCOL

The imaging control of intracranial aneurysms postembolization was represented by conventional angiography. Flow-up angiography was performed during the early years of our endovascular activities, and was completed by MR angiography in the later years. Thus, in case of a completely embolized aneurysm conventional cerebral angiography, Dyna$\mathrm{CT}$ and magnetic resonance angiography with threedimensional reconstruction (3D MRA) were recommended at $1.5,3,6,12$ months, postembolization, respectively. When a coil mass compaction, change configuration or migration was suspected in noninvasive studies, conventional angiography was performed immediately to check the exact state of the aneurysm and to decide the necessity of further treatment. If a stable occlusion is documented angiographically, follow-up conventional angiography was recommended after another 12 to 36 months. If stable occlusion was confirmed in post-embolization angiography performed after 1 to 2 years, follow-up was continued by noninvazive imaging studies $[1,2]$.

In cases with unsatisfactory (low grade of coil packing) or incomplete aneurysm occlusion after initial coil embolization, we performed control conventional angiography at 3 months postembolization. The next follow-up protocol depended on the stability of the coil mass and configuration of the embolized aneurysm. If a minor aneurysmal recanalization by minimal coil compaction at the aneurysmal neck occurs during the follow up, another 6-month MRA and 1-year angiography is performed. Instead, an early additional embolization treatment we performed when a major recanalization, with significant coil loosening, coil compaction or coil mass extrusion beyond the edge of a coil basket, or contrast filling within an aneurysm sac, occurs [2,3].

\section{DISCUSSION}

Most of the largest studies from literature demonstrated that in patients treated for ruptured cerebral aneurysm, the risk of recurrence and rebleeding is significantly higher with endovascular coil embolization in comparison with surgery. Also, a direct relationship was shown between endovascular treatment and/or aneurysmal rupture, with an increased rate of incomplete occlusion of aneurysms.

The aneurismal recurrence was defined in the literature as being residual larger than $20 \%$ of the primary aneurysm, unstable progressing neck 
remnants, aneurysm regrowth with or without coil compactation, and outgrowth of new aneurysmal sac. The evaluation of aneurysmal recurrence was usually performed by angioarchitecture comparing of angiographic controls with immediate postcoiling angiograms based on aneurysm sac size, neck morphology, dome-to-neck ratio and initial coil packing. Dorfer and all showed that $15,2 \%$ of the aneurysm smaller than $10 \mathrm{~mm}$ and $38,2 \%$ of the aneurysm larger than $10 \mathrm{~mm}$ had relevant recurrences and similarly $17,2 \%$ of aneurysm with neck $<4 \mathrm{~mm}$ and $27,3 \%$ with neck $>4 \mathrm{~mm}$ presented with recurrences. They also reported that a threshold of $25 \%$ packing density and 30\% volumetric aneurysm filling has been correlated with significantly stable angiographic results $[2,5,6]$.

Different mechanisms of aneurysm recurrence after endovascular treatment were described in large series. The most common mechanism of aneurysmal recurrence is residual aneurysm due to coils compaction. This is the most common situation in the cases of large and giant aneurysms with simple coils embolization. Lower rates of repermebilisation were reported for those aneurysms when the stentassisted or balloon technique was associated with coil occlusion. Coil migration into the intraluminal thrombus was also frequently reported especially in large partial thrombosed aneurysm. If initially this situation was considered by specialists as a separate entity, it is currently included in the category of compaction aneurysms. Nevertheless, coil mass compactation was not constantly associated with aneurysm recurrence. There have been many situations where the coil compaction phenomenon has been stable over time without any evolution to an aneurysm recurrence that would impose a type of reintervention $[1,2,3]$.
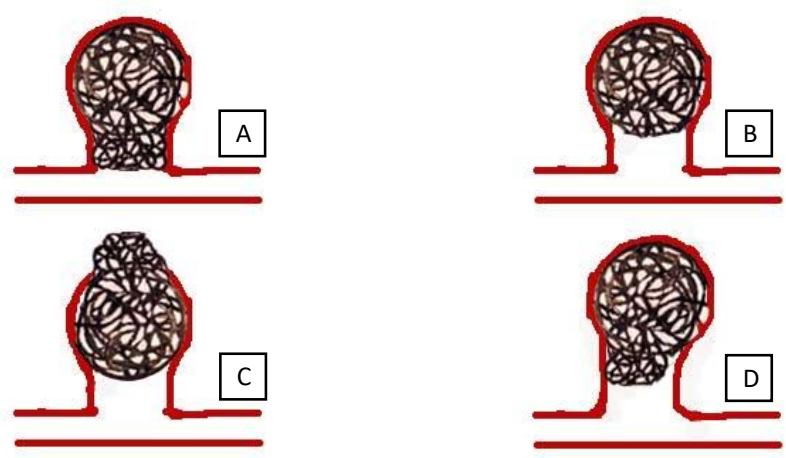

FIGURE 2: Mechanisms of aneurysm recurrence: A-Coil complete occlusion; B-Coil compactation; C-Coil Fundal migration; D-Coil regrowth.
In the situations of aneurysmal enlargement without signs of coil compactation, the mechanisms of aneurysm recurrence were defined as regrowth.

The third entity of aneurysmal recurrence mechanism defined by the last publications was fundal migration of the coil package through the wall of the aneurysmal sac. Also, some authors have mentioned as an aneurysm recurrence mechanism a combination of regrowth and fundal migration or compactation and fundal migration. All of these situations imposed an additional treatment.

Aneurysm recurrence could benefit from both endovascular and surgical treatment. The decision, regarding which type of treatment must be used, should be individualized as much as possible for each individual case. Most of the studies demonstrate that additional treatment is usually associated with a low complication risk and results in satisfactory stable occlusion in most patients. The main indication for initiating additional treatment was made because of the reported increased risk of further haemorrhage associated with unstable remnants and incompletely occluded aneurysms. The reported rebleed rate after endovascular therapy indicated in ISAT1 was $0.2 \%$ per patient year with a follow-up from 1 to 8 years (mean, 4 years) and $1.3 / 100$ patient years in the CARAT study, with no haemorrhage occurred after 2 years[..]. The main factors to be considered when deciding on the method of treatment used in the aneurysmal recurrence were represented by the aneurysm size and location, patient age and clinical condition, size of aneurysm remnant and its relation with adjacent vessels, presence of coils in the aneurysm neck, mechanism of recurrence and history of SAH manifestation $[2,6]$.

The time interval between aneurysm coil occlusion and angiographic control that demonstrate a clear evolution to aneurysm recurrence which require additional occlusion treatment was named the recurrence latency. It was demonstrated that the first control angiogram often performed at approximately 6 months is not sufficient to detect all aneurysms that may require additional treatment. Raymond et al have also reported progressive deterioration with aneurysms recurrences that were angiographically occluded at 6 months. Progressive neck remnant enlargement has been demonstrated in $14.8 \%$ of aneurysms in the first year after treatment and a major recurrence in 
$20.7 \%$ at a mean of $16.48 \pm 15.93$ months. The other authors detected $46.9 \%$ of all recurrences by 6 months and $96.9 \%$ by 36 months $[1,2,5]$.

The complication rate following aneurysms retreatment by endovascular retreatment ranged from $0 \%$ to $11 \%$ in previous reported series. Some of these series included patients with multistage treatment. Thromboembolic events were the major complication mentioned but only $3.2 \%$ of patients had a permanent neurologic deficit as Henkes et al described [2].

\section{CONCLUSIONS}

The coil mass instability due to compactation, migration or aneurysm regrowth that impose additional retreatment remains a major deficiency of endovascular treatment. The great challenge in managing patients with aneurysm recurrences remain the ability to early recognition of unstable aneurysm residuals with rehemorrhage potential by benign nonprogresive one. In case of relevant aneurysm recurrences both endovascular and surgical techniques could be successfully applied.

\section{REFERENCES}

1. Boet, R., Poon, W. S., \& Yu, S. C. H. (2001). The Management of Residual and Recurrent Intracranial Aneurysms After Previous Endovascular or Surgical Treatment-A Report of Eighteen Cases. Annals of The College of Surgeons of Hong Kong, 5(1), A3-A3.

2. Dorfer, C., Gruber, A., Standhardt, H., Bavinzski, G., \& Knosp, E. (2011). Management of residual and recurrent aneurysms after initial endovascular treatment. Neurosurgery, 70(3), 537-554.

3. Liu, J. J., Nielsen, T. H., Abhinav, K., Lee, J., Han, S. S., Marks, M. P., ... \& Steinberg, G. K. (2019). Surgical Treatment of Recurrent Previously Coiled and/or Stent-Coiled Intracerebral Aneurysms: A Single-Center Experience in a Series of 75 Patients. World neurosurgery.

4. Renowden, S. A., Koumellis, P., Benes, V., Mukonoweshuro, W., Molyneux, A. J., \& McConachie, N. S. (2008). Retreatment of previously embolized cerebral aneurysms: the risk of further coil embolization does not negate the advantage of the initial embolization. American Journal of Neuroradiology, 29(7), 1401-1404.

5. Sedat, J., Chau, Y., Moubarak, K., Vargas, J., \& Lonjon, M. (2012). Endovascular treatment of recurrent coiled aneurysms: assessment of complications and rebleeding during a decade in a single center. Interventional Neuroradiology, 18(1), 14-19.

6. Yu, L. B., Fang, Z. J., Yang, X. J., \& Zhang, D. (2019). Management of Residual and Recurrent Aneurysms After Clipping or Coiling: Clinical Characteristics, Treatments, and Follow-Up Outcomes. World neurosurgery, 122, e838e846. 